\title{
SCIENTIFIC REPORTS

\section{The majority of autosomal recessive nanophthalmos and posterior microphthalmia can be attributed to biallelic sequence and structural variants in MFRP and PRSS56}

\author{
Basamat Almoallem $\mathbb{1}^{1,2}$, Gavin Arno ${ }^{3,4}$, Julie De Zaeytijd ${ }^{5}$, Hannah Verdin ${ }^{1}$, Irina Balikova ${ }^{5}$, \\ Ingele Casteels ${ }^{6}$, Thomy de Ravel ${ }^{7}$, Sarah Hull ${ }^{3,4}$, Martina Suzani ${ }^{3,4}$, Anne Destrée ${ }^{8}$, \\ Michelle Peng ${ }^{9}$, Denise Williams ${ }^{10}$, John R. Ainsworth ${ }^{10}$, Andrew R. Webster ${ }^{3,4}$, \\ Bart P. Leroy ${ }^{1,5,11}$, Anthony T. Moore ${ }^{3,4,9}$ \& Elfride De Baere $\mathbb{1}^{1 *}$
}

This study aimed to genetically and clinically characterize a unique cohort of 25 individuals from 21 unrelated families with autosomal recessive nanophthalmos (NNO) and posterior microphthalmia (MCOP) from different ethnicities. An ophthalmological assessment in all families was followed by targeted MFRP and PRSS56 testing in 20 families and whole-genome sequencing in one family. Three families underwent homozygosity mapping using SNP arrays. Eight distinct MFRP mutations were found in 10/21 families (47.6\%), five of which are novel including a deletion spanning the $5^{\prime}$ untranslated region and the first coding part of exon 1 . Most cases harbored homozygous mutations (8/10), while a compound heterozygous and a monoallelic genotype were identified in the remaining ones (2/10). Six distinct PRSS56 mutations were found in 9/21 (42.9\%) families, three of which are novel. Similarly, homozygous mutations were found in all but one, leaving 2/21 families (9.5\%) without a molecular diagnosis. Clinically, all patients had reduced visual acuity, hyperopia, short axial length and crowded optic discs. Retinitis pigmentosa was observed in 5/10 (50\%) of the MFRP group, papillomacular folds in $12 / 19(63.2 \%)$ of MCOP and in 3/6 (50\%) of NNO cases. A considerable phenotypic variability was observed, with no clear genotype-phenotype correlations. Overall, our study represents the largest NNO and MCOP cohort reported to date and provides a genetic diagnosis in 19/21 families (90.5\%), including the first MFRP genomic rearrangement, offering opportunities for gene-based therapies in MFRP-associated disease. Finally, our study underscores the importance of sequence and copy number analysis of the MFRP and PRSS56 genes in MCOP and NNO.

Congenital microphthalmia (MCO) is a heterogeneous developmental eye disease characterized by small, hyperopic eyes secondary to several etiological factors affecting the early formation of the optic cup ${ }^{1-3}$. MCO has a reported incidence of approximately 15/100,000 live births per year ${ }^{4}$ and may be isolated, complex or syndromic based on the presence or absence of associated ocular malformations and systemic involvement ${ }^{2,5}$. Moreover,

\footnotetext{
${ }^{1}$ Center for Medical Genetics, Ghent University and Ghent University Hospital, Ghent, Belgium. ${ }^{2}$ Department of Ophthalmology, King Abdul-Aziz University Hospital, College of Medicine, King Saud University, Riyadh, Saudi Arabia. ${ }^{3}$ Institute of Ophthalmology, University College London, London, United Kingdom. ${ }^{4}$ Department of Ophthalmology, Moorfields Eye Hospital, London, United Kingdom. ${ }^{5}$ Department of Ophthalmology, Ghent University and Ghent University Hospital, Ghent, Belgium. ${ }^{6}$ Department of Ophthalmology, University Hospitals Leuven, Leuven, Belgium. ${ }^{7}$ Center for Human Genetics, University Hospitals Leuven, Leuven, Belgium. ${ }^{8}$ Department of Human Genetics, Institut de Pathologie et de Génétique (IPG), Charleroi, Belgium. ${ }^{9}$ Department of Ophthalmology, University of California, San Francisco, California, United States. ${ }^{10}$ Birmingham Women's NHS Foundation Trust, Birmingham, UK. ${ }^{11}$ Division of Ophthalmology, The Children's Hospital of Philadelphia, Philadelphia, Pennsylvania, United States. *email: elfride.debaere@ugent.be
} 
MCO can be subclassified into two clinical subtypes called nanophthalmos (NNO) and posterior microphthalmia (MCOP), based on the involvement (NNO) or not (MCOP) of the anterior segment $t^{6,7}$. Both NNO and MCOP may be associated with retinitis pigmentosa (RP), foveoschisis and optic disc drusen ${ }^{8-10}$.

Clinically both conditions are characterized by a short axial length that gives rise to high hyperopia ranging between +8.00 to +25.00 diopters ${ }^{11}$. Best-corrected visual acuity (BCVA) is reduced and rarely better than $20 / 40^{12-15}$. This poor BCVA is primarily caused by the hyperopia but may be aggravated by posterior segment changes such as an abnormal foveal structure and so-called papillomacular folds, uveal effusion, and an abnormal foveal avascular zone $e^{13,16}$. Patients with NNO or MCOP are prone to complications such as angle closure glaucoma, uveal effusion after intra-ocular surgery, non-rhegmatogenous retinal detachment and intraretinal cysts $^{13,16-18}$.

Biallelic mutations in the MFRP (encoding membrane-type frizzled related protein, MIM 606227) or PRSS56 (encoding protease serine 56, MIM 613858) genes have been reported to cause autosomal recessive NNO or MCOP. MFRP ${ }^{19,20}$ was found to be expressed predominantly in the retinal pigment epithelium (RPE) and ciliary epithelium of the eye, with a weak expression in fetal brain ${ }^{3}$. Mouse and zebrafish studies confirmed its expression in the RPE and ciliary body ${ }^{20}$. Zebrafish models recapitulated reduced axial length causing hyperopia, reduced visual acuity and RPE folding ${ }^{21}$. Apart from its role in eye development and emmetropization, MFRP plays a role in photoreceptor maintenance, explaining the risk for RP-like changes when mutated ${ }^{8,22,23}$. PRSS56 was found to be expressed in human neural retina, cornea, sclera, and the optic nerve. Mouse Prss56 is expressed in the eye from embryonic development until adult life ${ }^{17}$. Mouse studies and genome-wide association studies suggest that PRSS56 is taking part of a regulatory network influencing postnatal eye development and emmetropization ${ }^{24-26}$.

Here, we aimed to genetically and clinically characterize 25 individuals from 21 unrelated families with autosomal recessive NNO or MCOP from different ethnicities, representing the largest cohort reported to date. This revealed eight distinct $M F R P$ and six distinct PRSS56 mutations respectively, providing a molecular diagnosis in 19/21 families (90.5\%) and uncovering opportunities for gene-based therapies in MFRP-associated disease.

\section{Methods}

Patients and clinical assessment. Twenty-five patients out of 21 unrelated families from different ethnicities with either isolated or complex NNO or MCOP were recruited from three centers: Ghent University Hospital, Belgium ( $\mathrm{n}=11)$; Moorfields Eye Hospital, London, UK $(\mathrm{n}=7)$; and Birmingham Children's Hospital, Birmingham, UK $(n=7)$ (Table 1). A full ophthalmological examination included BCVA measurement and dilated fundus examination. Retinal fundus imaging was obtained by conventional 30-degree fundus colour photographs (Topcon Great Britain Ltd, Berkshire, UK) or via ultra-wide field confocal scanning laser imaging (Optos plc, Dunfermline, UK), near-infrared and blue light fundus autofluorescence (FAF) imaging (Spectralis, Heidelberg Engineering Ltd, Heidelberg, Germany), and spectral domain optical coherence tomography (OCT) scans (Spectralis, Heidelberg Engineering Ltd, Heidelberg, Germany). Full-field electroretinography (ERG) was performed according to the International Society for Clinical Electrophysiology of Vision (ISCEV) standards ${ }^{27}$ (using either Roland Consult, Brandenburg an den Havel, Germany or Diagnosys, Cambridge, UK equipment). Genomic DNA was extracted from EDTA blood using standard procedures. This study was conducted following the tenets of the Declaration of Helsinki.

Homozygosity mapping. Homozygosity mapping using genome-wide single-nucleotide polymorphisms (SNP) arrays was performed in three patients originating from a self-reported consanguineous marriage, using HumanCytoSNP-12 BeadChips (Illumina, San Diego, CA). Homozygous regions ( $>1 \mathrm{Mb}$ ) were identified using $\mathrm{PLINK}^{28}$ software integrated in $\mathrm{ViVar}^{29}$. Resulting homozygous regions were ranked according to their length and number of SNPs, as described ${ }^{30}$.

Mutation screening by Sanger sequencing and by whole genome sequencing. Primers for PCR amplification of the coding region and splice site junctions of MFRP and PRSS56 were designed (available upon request). Sanger sequencing was performed according to the manufacturer's instructions (BigDyeTerminator v3.1 Cycle Sequencing Kit, ABI 3730XL genetic analyzer, Thermo Fisher Scientific, Waltham, MA).

One family (F7.GC20271) was included in a whole genome sequencing (WGS) study. Genome enrichment was performed using the Illumina TruSeq DNA PCR-Free Sample preparation kit (Illumina, Inc.), followed by sequencing on an Illumina HiSeq 2500 with a minimum coverage of $15 x$ for approximately $95 \%$ of the genome. The Isaac Genome Alignment Software (version 01.14; Illumina, Inc.) was used for reads mapping against the Genome Reference Consortium human genome build 37 (GRCh37) ${ }^{31}$. Standard variant filtering was performed as previously described ${ }^{32,33}$. Structural variant (SV) assessment was done by interrogation of copy number variation $(\mathrm{CNV})$, SV calls and visual inspection of the individual split and chimeric reads (Integrated Genome Viewer, IGV) across the breakpoints as described by Carss et al. ${ }^{32}$. A genomic rearrangement found in MFRP (GRCh37 [hg19] chr11:119,217, 130_119, 223, 310delinsACCACTA, NM_031433.3) was confirmed using a junction PCR followed by Sanger sequencing of the junction product and by characterization of the breakpoint junctions.

Variant interpretation. Variant classification was performed following ACMG guidelines ${ }^{34}$. Using Alamut Visual (v. 2.7) (Interactive Biosoftware, Rouen, France), following in silico prediction tools were used: Align GVGD, Sorting Intolerant From Tolerant [SIFT], MutationTaster, and PolyPhen-2, Grantham score calculation, conservation. Several genomic databases including dbSNP build 145 (http://www.ncbi.nlm.nih.gov/SNP/) and gnomAD (http://gnomad.broadinstitute.org) were used to assess variant frequencies in a general population. Segregation analysis was performed in all available family members. Mutation nomenclature uses numbering with the A of the initiation codon ATG as +1 (http://varnomen.hgvs.org/) based on the following RefSeqs: NM_031433.3 (MFRP) and NM_001195129.1 (PRSS56). 


\begin{tabular}{|c|c|c|c|c|c|c|c|c|c|c|c|c|c|c|c|c|c|c|c|c|c|}
\hline \multirow[b]{2}{*}{ FA\# } & \multirow[b]{2}{*}{ PT \# } & \multirow[b]{2}{*}{\begin{tabular}{|l} 
Extended \\
ID
\end{tabular}} & \multirow[b]{2}{*}{ Sex } & \multirow[b]{2}{*}{$\begin{array}{l}\text { Ethnicity/ } \\
\text { Consanguinity }\end{array}$} & \multirow[b]{2}{*}{$\begin{array}{l}\text { Age } \\
\text { (year) }\end{array}$} & \multirow[b]{2}{*}{ Group } & \multirow[b]{2}{*}{ Gene } & \multicolumn{2}{|c|}{$\begin{array}{l}\text { BCVA, } \\
\text { logMAR } \\
\text { (Snellen) }\end{array}$} & \multicolumn{5}{|c|}{ Posterior segment features } & \multicolumn{2}{|c|}{$\mathbf{A L}(\mathbf{m m})$} & \multicolumn{2}{|c|}{\begin{tabular}{|l|} 
Posterior \\
coat \\
thickness \\
$(\mathrm{mm})$
\end{tabular}} & \multirow[b]{2}{*}{$\begin{array}{l}\text { Refraction } \\
\text { (diopter) }\end{array}$} & \multirow[b]{2}{*}{ ERG } & \multirow[b]{2}{*}{ Diagnosis } \\
\hline & & & & & & & & $\mathbf{R}$ & $\mathbf{L}$ & $\begin{array}{l}\text { Crow- } \\
\text { ded } \\
\text { discs }\end{array}$ & \begin{tabular}{|l|}
$\begin{array}{l}\text { Papillo- } \\
\text { macular } \\
\text { fold }\end{array}$ \\
\end{tabular} & $\begin{array}{l}\text { Macular } \\
\text { edema }\end{array}$ & $\begin{array}{l}\text { White } \\
\text { dots }\end{array}$ & \begin{tabular}{|l|} 
Perip- \\
heral \\
pigment
\end{tabular} & $\mathbf{R}$ & $\mathbf{L}$ & $\mathbf{R}$ & $\mathbf{L}$ & & & \\
\hline F1 & P1 & B03271 & F & $\begin{array}{l}\text { Belgian/ } \\
\text { Consanguineous }\end{array}$ & 23 & Ghent & $M F R P$ & 0.6 & 0.3 & + & + & + & + & + & 16.1 & 15.7 & NA & NA & \begin{tabular}{|l|}
$\mathrm{R}+14,00 /$ \\
$-0,50 \times 13$ \\
$\mathrm{~L}+15,50 /$ \\
$-0,25 \times 117$
\end{tabular} & SN & $\mathrm{NNO} / \mathrm{RP}$ \\
\hline $\mathrm{F} 2$ & P2 & B14785 & M & $\begin{array}{l}\text { Moroccan/ } \\
\text { Consanguineous }\end{array}$ & 3 & Ghent & MFRP & 0.4 & 0.5 & + & + & - & + & - & 14.5 & 14.4 & NA & NA & \begin{tabular}{|l|}
$\mathrm{R}+12.00 /$ \\
$+1.00 \times 180$ \\
$\mathrm{~L}+12.00 /$ \\
$+1.00 \times 180$
\end{tabular} & SN & MCOP \\
\hline F3 & P3 & GC19623 & $\mathrm{F}$ & Indian/NA & 29 & London & $M F R P$ & 0.5 & 0.5 & + & - & + & - & - & 13.3 & 12.8 & 2.1 & 2.1 & \begin{tabular}{|l|}
$\mathrm{R}+15.25 /$ \\
$-0.25 \times 175$ \\
$\mathrm{~L}+15.25 /$ \\
$-0.50 \times 155$
\end{tabular} & NL & MCOP \\
\hline $\mathrm{F} 4$ & P4 & GC19691 & F & $\begin{array}{l}\text { British } \\
\text { Caucasian/NA }\end{array}$ & 15 & London & $M F R P$ & 0.2 & 0.3 & + & + & - & + & + & 15.5 & 15.5 & 2.1 & 2.1 & $\begin{array}{l}\mathrm{R}+16.00 / \\
-0.5 \times 50 \\
\mathrm{~L}+16.00 / \\
-0.5 \times 100\end{array}$ & SN & MCOP \\
\hline F5 & P5 & B09352 & F & $\begin{array}{l}\text { Dagestanian/ } \\
\text { Consanguineous }\end{array}$ & 68 & Ghent & MFRP & 0.3 & 0.1 & + & - & + & + & + & 16.5 & 16.5 & NA & NA & \begin{tabular}{|l|}
$\mathrm{R}+8,50 /$ \\
$-1,00 \times 106$ \\
$\mathrm{~L}+8,75 /$ \\
$-0,75 \times 113$
\end{tabular} & NR & NNO/RP \\
\hline F6 & P6 & GC18886 & M & Kurdish/NA & 31 & London & $M F R P$ & 0.8 & 0.6 & + & - & + & - & + & 16.5 & 16.3 & 1.7 & 1.9 & $\begin{array}{l}\mathrm{R}+17.5 / \\
-0.50 \times 95 \\
\mathrm{~L}+16.00 / \\
-0.50 \times 90\end{array}$ & NA & MCOP/RP \\
\hline F7 & P7 & GC20271 & F & Iranian/NA & 51 & London & $M F R P$ & 0.6 & 1.3 & + & - & + & - & + & NA & NA & NA & NA & \begin{tabular}{|l|}
$\mathrm{R}+15.0 /$ \\
$-1.00 \times 100$ \\
$\mathrm{~L}+15.5 /$ \\
$-0.75 \times 110$
\end{tabular} & SN & $\mathrm{MCOP} / \mathrm{RP}$ \\
\hline F8 & P8 & B10315 & M & Italian/NA & 26 & Ghent & $M F R P$ & LP & LP & + & - & + & + & - & 15.7 & 15.7 & NA & NA & \begin{tabular}{|l|}
$\mathrm{R}+15.25 /$ \\
$-0.25 \times 175$ \\
$\mathrm{~L}+15.25 /$ \\
$-0.50 \times 155$
\end{tabular} & SN & $\mathrm{NNO} / \mathrm{RP}$ \\
\hline F9 & P9 & B16796 & F & Belgian/NA & 75 & Ghent & $M F R P$ & 0.1 & 0.1 & + & - & + & + & + & NA & NA & NA & NA & \begin{tabular}{|l|}
$\mathrm{R}+17,00 /$ \\
$0,75 \times 15$ \\
$\mathrm{~L}+15,00 /$ \\
$-0,75 \times 170$
\end{tabular} & SN & MCOP \\
\hline F10 & P10 & B08047 & M & $\begin{array}{l}\text { Moroccan/ } \\
\text { Consanguineous }\end{array}$ & 8 & Ghent & $M F R P$ & 0.25 & 0.25 & + & - & NA & NA & NA & NA & NA & $\mathrm{NA}$ & NA & $\begin{array}{l}\mathrm{R}+13.75 \\
\mathrm{~L}+13.25\end{array}$ & NA & NNO \\
\hline F11 & P11 & GC20258 & F & $\begin{array}{l}\text { British } \\
\text { Caucasian/NA }\end{array}$ & 18 & London & PRSS56 & 0.6 & 0.3 & + & - & - & + & - & 15.7 & 15.8 & 2.3 & 2.1 & \begin{tabular}{|l|}
$\mathrm{R}+15.00 /$ \\
$-0.5 \times 180$ \\
$\mathrm{~L}+13.50 /$ \\
$-0.75 \times 180$
\end{tabular} & NL & MCOP \\
\hline F12 & P12 & GC18588 & M & Pakistani/NA & 37 & London & PRSS56 & 0.5 & 0.6 & + & + & + & + & - & 15.4 & 15.4 & 1.9 & 1.9 & \begin{tabular}{|l|}
$\mathrm{R}+2 /$ \\
$-1.25 \times 180$ \\
$\mathrm{~L}+4.5 /$ \\
$-1.5 \times 180$
\end{tabular} & $\mathrm{SN}$ & MCOP \\
\hline F13 & P13 & GC16899 & M & Somalian/NA & 20 & London & PRSS56 & 0.6 & 0.6 & + & + & + & + & - & 16.5 & 16.4 & 1.9 & 2.1 & \begin{tabular}{|l|}
$\mathrm{R}+15.00 /$ \\
$-0.75 \times 110$ \\
$\mathrm{~L}+15.50 /$ \\
$-0.50 \times 75$
\end{tabular} & SN & MCOP \\
\hline F14 & P14 & GC19721 & M & Pakistani/NA & 23 & London & PRSS56 & 0.8 & 0.8 & + & + & - & + & - & 15.9 & 15.7 & 2.1 & 2.1 & $\begin{array}{l}\mathrm{R}+9.50 / \\
-1.00 \times 20 \\
\mathrm{~L}+11.00 / \\
-1.00 \times 40\end{array}$ & SN & MCOP \\
\hline \multirow{5}{*}{ F15 } & P15 & \begin{tabular}{|l|} 
RXK- \\
4624916 \\
\end{tabular} & F & $\begin{array}{l}\text { Pakistani/ } \\
\text { Consanguineous }\end{array}$ & 44 & \begin{tabular}{|l|}
$\begin{array}{l}\text { Birmin- } \\
\text { gham }\end{array}$ \\
\end{tabular} & PRSS56 & NA & NA & + & + & - & - & + & 17 & 17 & 2.4 & 2.4 & $\begin{array}{l}\mathrm{R}+15.00 \\
\mathrm{~L}+15.50\end{array}$ & NA & MCOP/RP \\
\hline & P16 & \begin{tabular}{|l|} 
RXK- \\
3297132 \\
\end{tabular} & M & $\begin{array}{l}\text { Pakistani/ } \\
\text { Consanguineous }\end{array}$ & 13 & \begin{tabular}{|l|}
$\begin{array}{l}\text { Birmin- } \\
\text { gham }\end{array}$ \\
\end{tabular} & PRSS56 & 0.2 & 0.1 & + & + & - & + & + & 15.2 & 15.6 & NA & NA & $\begin{array}{l}\mathrm{R}+14.00 \\
\mathrm{~L}+14.50\end{array}$ & NL & MCOP/RP \\
\hline & P17 & \begin{tabular}{|l|} 
RXK- \\
33447157
\end{tabular} & F & $\begin{array}{l}\text { Pakistani/ } \\
\text { Consanguineous }\end{array}$ & 8 & \begin{tabular}{|l|}
$\begin{array}{l}\text { Birmin- } \\
\text { gham }\end{array}$ \\
\end{tabular} & PRSS56 & 0.6 & 0.6 & + & - & - & + & - & 14.0 & 14.2 & NA & NA & $\begin{array}{l}\mathrm{R}+16.00 \\
\mathrm{~L}+18.50 / \\
-1.00 \times 10\end{array}$ & SN & MCOP \\
\hline & P18 & $\begin{array}{l}\text { RXK- } \\
3113374\end{array}$ & F & $\begin{array}{l}\text { Pakistani/ } \\
\text { Consanguineous }\end{array}$ & 12 & $\begin{array}{l}\begin{array}{l}\text { Birmin- } \\
\text { gham }\end{array} \\
\end{array}$ & PRSS56 & 0.5 & 0.5 & + & + & - & - & - & 14.8 & 14.6 & NA & NA & $\begin{array}{l}\mathrm{R}+15.50 / \\
-1.50 \times 30 \\
\mathrm{~L}+14.50 / \\
-1.00 \times 8\end{array}$ & SN & MCOP \\
\hline & P19 & \begin{tabular}{|l|} 
RXK- \\
4633376
\end{tabular} & F & $\begin{array}{l}\text { Pakistani/ } \\
\text { Consanguineous }\end{array}$ & 17 & \begin{tabular}{|l|} 
Birmin- \\
gham
\end{tabular} & PRSS56 & 0.5 & 0.5 & + & - & - & - & - & 14.5 & 14.5 & NA & NA & \begin{tabular}{|l|}
$\mathrm{R}+16.00 /$ \\
$-1.00 \times 180$ \\
$\mathrm{~L}+15.00 /$ \\
$-1.00 \times 180$
\end{tabular} & NL & MCOP \\
\hline F16 & P20 & \begin{tabular}{|l|} 
RXK- \\
4941281
\end{tabular} & M & $\begin{array}{l}\text { Mirpuri/ } \\
\text { Consanguineous }\end{array}$ & 7 & \begin{tabular}{|l|} 
Birmin- \\
gham
\end{tabular} & PRSS56 & 0.5 & 0.1 & + & + & - & - & - & 16.0 & 16.2 & 2.4 & 2.4 & $\begin{array}{l}\mathrm{R}+16.25 / \\
-1.00 \times 180 \\
\mathrm{~L}+17.25 / \\
-1.75 \times 180\end{array}$ & NA & MCOP \\
\hline F17 & P21 & \begin{tabular}{|l|} 
RXK- \\
4985235
\end{tabular} & F & $\begin{array}{l}\text { Mirpuri/ } \\
\text { Consanguineous }\end{array}$ & 10 & \begin{tabular}{|l|}
$\begin{array}{l}\text { Birmin- } \\
\text { gham }\end{array}$ \\
\end{tabular} & PRSS56 & 0.5 & 0.5 & + & + & - & - & - & 15.0 & 15.1 & 2.8 & 2.7 & $\begin{array}{l}\mathrm{R}+19.50 \\
\mathrm{~L}+19.50\end{array}$ & NA & MCOP \\
\hline
\end{tabular}




\begin{tabular}{|c|c|c|c|c|c|c|c|c|c|c|c|c|c|c|c|c|c|c|c|c|c|}
\hline \multirow[b]{2}{*}{ FA\# } & \multirow[b]{2}{*}{$\mathbf{P T} \#$} & \multirow[b]{2}{*}{$\begin{array}{l}\text { Extended } \\
\text { ID }\end{array}$} & \multirow[b]{2}{*}{ Sex } & \multirow[b]{2}{*}{$\begin{array}{l}\text { Ethnicity/ } \\
\text { Consanguinity }\end{array}$} & \multirow[b]{2}{*}{$\begin{array}{l}\text { Age } \\
\text { (year) }\end{array}$} & \multirow[b]{2}{*}{ Group } & \multirow[b]{2}{*}{ Gene } & \multicolumn{2}{|c|}{$\begin{array}{l}\text { BCVA, } \\
\text { logMAR } \\
\text { (Snellen) }\end{array}$} & \multicolumn{5}{|c|}{ Posterior segment features } & \multicolumn{2}{|c|}{$\mathbf{A L}(\mathbf{m m})$} & \multicolumn{2}{|c|}{$\begin{array}{l}\text { Posterior } \\
\text { coat } \\
\text { thickness } \\
(\mathrm{mm})\end{array}$} & \multirow[b]{2}{*}{$\begin{array}{l}\text { Refraction } \\
\text { (diopter) }\end{array}$} & \multirow[b]{2}{*}{ ERG } & \multirow[b]{2}{*}{ Diagnosis } \\
\hline & & & & & & & & $\mathbf{R}$ & $\mathbf{L}$ & $\begin{array}{l}\text { Crow- } \\
\text { ded } \\
\text { discs }\end{array}$ & $\begin{array}{l}\text { Papillo- } \\
\text { macular } \\
\text { fold }\end{array}$ & $\begin{array}{l}\text { Macular } \\
\text { edema }\end{array}$ & $\begin{array}{l}\text { White } \\
\text { dots }\end{array}$ & $\begin{array}{l}\text { Perip- } \\
\text { heral } \\
\text { pigment }\end{array}$ & $\mathbf{R}$ & $\mathbf{L}$ & $\mathbf{R}$ & $\mathbf{L}$ & & & \\
\hline F18 & P22 & B05802 & $\mathrm{M}$ & $\begin{array}{l}\text { Turkish/ } \\
\text { Consanguineous }\end{array}$ & 4 & Ghent & PRSS56 & 0.3 & 0.3 & + & + & + & - & - & 15.3 & 15.1 & NA & NA & $\begin{array}{l}\mathrm{R}+14,25 / \\
-0,50 \times 4 \\
\mathrm{~L}+15,00 / \\
-0,50 \times 174\end{array}$ & NL & MCOP \\
\hline F19 & $\mathrm{P} 23$ & B01921 & M & Bulgarian/NA & 26 & Ghent & PRSS56 & 0.4 & 0.5 & + & + & + & - & - & 14.8 & 14.6 & NA & NA & $\begin{array}{l}\mathrm{R}+15.25 / \\
-0.25 \times 175 \\
\mathrm{~L}+15.25 / \\
-0.50 \times 155\end{array}$ & SN & NNO \\
\hline F20 & P24 & B03421 & M & Belgian/NA & 7 & Ghent & I & 0.3 & 0.4 & + & + & + & - & - & 14.5 & 14.5 & $\mathrm{NA}$ & NA & $\begin{array}{l}\mathrm{R}+14,25 / \\
-1.00 \times 13 \\
\mathrm{~L}+15,25 / \\
-1.75 \times 173\end{array}$ & NL & MCOP \\
\hline F21 & P25 & B07457 & $\mathrm{M}$ & Belgian/NA & 8 & Ghent & I & 0.2 & 0.2 & + & + & NA & NA & NA & NA & $\mathrm{NA}$ & $\mathrm{NA}$ & NA & $\begin{array}{l}\text { R NA } \\
\text { L NA }\end{array}$ & NA & NNO \\
\hline
\end{tabular}

Table 1. Clinical features of patients from 21 unrelated families with isolated or complex NNO or MCOP. Abbreviations used: FA\#: family number; PT\#: patient number; NR: not-reported; AL: axial length; BCVA: best-corrected visual acuity; ERG: electroretinogram; F: female; L: left eye; M: male; mm: millimeters; NA: not available; NL: normal limit; NR: non-recordable; R: right eye; SN: subnormal; “/”: no mutation identified; +: presence; -: absence; MCOP: isolated posterior microphthalmia; MCOP/RP: posterior microphthalmia with $\mathrm{RP}$; NNO: isolated nanophthalmos; NNO/RP: nanophthalmos with RP; retinitis pigmentosa: RP.

\section{Results}

Novel and known variants in MFRP and PRSS56. Homozygosity mapping in three families with a reported consanguineous background revealed the presence of MFRP in homozygous regions of $10.2 \mathrm{Mb}$ and $6.2 \mathrm{Mb}$ in two families (F1 and F2) respectively, while PRSS56 was found in a homozygous region of $6.5 \mathrm{Mb}$ in the third family (F18) (Fig. S1). Subsequent testing of the MFRP and PRSS56 genes revealed three distinct homozygous mutations in these families, two of which are novel (Table 2). Direct testing of MFRP and PRSS56 in 17 families revealed 11 additional distinct mutations in MFRP and PRSS56, five of which are novel (Table 2).

WGS in one family (F7) revealed two novel heterozygous mutations in MFRP: a coding nonsense variant c. $955 \mathrm{C}>\mathrm{T}$ p. $\left(\mathrm{Gln} 319^{*}\right)$ and genomic rearrangement consisting of a deletion of $6.2 \mathrm{~kb}$ and an insertion of 7 nucleotides c. -6087 -54 +40delinsTAGTGGT p.(?). This genomic rearrangement encompasses the $5^{\prime} \mathrm{UTR}$ and the coding part of exon 1 and is predicted to abolish the transcription initiation site (Fig. 1A). The breakpoints of this deletion were characterized by a junction PCR followed by sequencing (Fig. 1B). An assessment of the breakpoints at the nucleotide level showed the insertion of seven base pairs (TAGTGGT), representing a potential information scar. As no microhomology was detected at the breakpoints and only one breakpoint overlapped with an Alu repeat, non-allelic homologous recombination (NAHR) and microhomology-based mechanisms are unlikely. Altogether, non-homologous end joining (NHEJ) is the most likely mechanism underlying this rearrangement ${ }^{35-37}$.

Overall, genetic defects were found in 19 of the 21 families (90.5\%): eight distinct MFRP mutations in ten families $(10 / 21,47.5 \%)$ and six distinct PRSS56 mutations in nine families $(9 / 21,42.9 \%)$. Biallelic mutations were found in 18 families, while a monoallelic MFRP mutation was found in one family, with an undiscovered second mutation. With mutations neither in MFRP nor PRSS56, two Belgian families $(2 / 21,9.5 \%)$ remained molecularly unaccounted for. A summary of all variants identified, their in silico assessment and ACMG variant classification can be found in Table 2. Flowchart of the molecular workflow and outcomes is provided in Fig. S2.

Phenotypic characteristics. Twenty-five patients from 21 unrelated families were investigated, six with a diagnosis of NNO and 19 with MCOP. The age of diagnosis varied from 3-75 years. Overall, all eyes had an axial length of $<18 \mathrm{~mm}$ and hyperopia of $>8$ diopters with crowded discs and foveal hypoplasia. The clinical features of all studied individuals are summarized in Table 1 . Based on the genotypes found, the families were divided into two genetic subtypes: a MFRP- and PRSS56-associated group.

$M F R P$ group. All affected individuals had reduced BCVA ranging from $0.1 \operatorname{logMAR}$ to light perception. All had crowded discs and loss of normal foveal architecture. In 3/10 (30\%) of the patients, there was evidence of papillomacular folds and intraretinal cysts. Moreover, peripheral retinal pigmentary changes were observed in 5/10 (50\%) patients, ranging from mild RPE hypopigmentation to extensive reticular hypopigmentation and occasional hyperpigmented lesions. ERG was performed on 8/10 (80\%) of MFRP-mutated patients, showing subnormal ERG responses for rod and cones in 6/8 (75\%) of the patients P1, P2, P4, P7, P8 and P9), a non-recordable ERG in 1/8 (12.5\%) (P5) and normal ERG responses in 1/8 (12.5\%) (P3). Representative retinal imaging of these individuals is shown in Fig. 2.

PRSS56 group. All affected individuals had reduced BCVA ranging from 0.1 to $0.8 \log$ MAR. Crowded discs, loss of normal foveal architecture and papillomacular folds were observed in 7/13 (53.8\%) patients. Peripheral retinal pigmentary changes were only found in two affected siblings (P15 and P16) $(2 / 13,15.4 \%)$. ERG was performed on 9/13 (69.2\%) patients, showing subnormal ERG recordings in 5/13 (71.4\%) (P12, P13, P14, P17 and 


\begin{tabular}{|c|c|c|c|c|c|c|c|c|c|c|c|c|c|c|c|}
\hline FAM. PID & Diagnosis & Gene & cDNA & Protein & $\begin{array}{l}\text { Geno- } \\
\text { type }\end{array}$ & Exon & Segregation & $\begin{array}{l}\text { Grantham } \\
\text { distance }\end{array}$ & SIFT & PolyPhen-2 & GVGD & $\begin{array}{l}\text { Mutation } \\
\text { Taster }\end{array}$ & \begin{tabular}{|l|} 
gnomAD \\
(Total \\
population \\
frequency)
\end{tabular} & $\begin{array}{l}\text { ACMG } \\
\text { Classifi- } \\
\text { cation }\end{array}$ & Reference \\
\hline $\begin{array}{l}\text { F1. } \\
\text { B03271 }\end{array}$ & NNO/RP & $M F R P$ & c.1090_1094del & $\begin{array}{l}\text { p.(Thr364 } \\
\text { Glnfs*26) }\end{array}$ & HOM & 9 & $\mathrm{NP}$ & I & 1 & I & 1 & 1 & $\begin{array}{l}0.0004102 \% \\
(0 \mathrm{HOM})\end{array}$ & Class 4 & This study \\
\hline $\begin{array}{l}\text { F2. } \\
\text { B14785 }\end{array}$ & MCOP & MFRP & c.498del & $\begin{array}{l}\text { p.(Asn167 } \\
\text { Thrfs*25) }\end{array}$ & HOM & 5 & Yes & I & 1 & I & 1 & I & \begin{tabular}{|l|}
$0.0004024 \%$ \\
$(0 \mathrm{HOM})$
\end{tabular} & Class 5 & $20,38,44,45,52$ \\
\hline $\begin{array}{l}\text { F3. } \\
\text { GC19623 }\end{array}$ & MCOP & MFRP & c. $1549 \mathrm{C}>\mathrm{T}$ & $\begin{array}{l}\text { p.(Arg517 } \\
\text { Trp) }\end{array}$ & ном & 13 & NP & 101 & $\mathrm{D}$ & $\begin{array}{l}\text { Prob. } \\
\text { dam. }\end{array}$ & $\mathrm{C} 25$ & $\begin{array}{l}\text { Dis. } \\
\text { caus. }\end{array}$ & \begin{tabular}{|l|}
$0.002022 \%$ \\
$(0 \mathrm{HOM})$
\end{tabular} & Class 4 & 41 \\
\hline $\begin{array}{l}\text { F4. } \\
\text { GC19691 }\end{array}$ & MCOP & MFRP & $\begin{array}{l}\text { c.491_492insT } \\
\text { 2nd variant } \\
\text { unknown }\end{array}$ & $\begin{array}{l}\text { p.(Asn167 } \\
\text { Glnfs*34) }\end{array}$ & HTZ & 5 & NP & I & 1 & I & 1 & 1 & $\begin{array}{l}0.005723 \% \\
(0 \mathrm{HOM})\end{array}$ & Class 5 & 36 \\
\hline $\begin{array}{l}\text { F5. } \\
\text { B09352 }\end{array}$ & $\begin{array}{l}\text { NNO/ } \\
\mathrm{RP}\end{array}$ & $M F R P$ & c.498dup & $\begin{array}{l}\text { p.(Asn167 } \\
\text { Glnfs*34) }\end{array}$ & HOM & 5 & $\mathrm{NP}$ & I & 1 & I & 1 & 1 & \begin{tabular}{|l|}
$\begin{array}{l}0.005723 \% \\
(0 \mathrm{HOM})\end{array}$ \\
\end{tabular} & Class 5 & $8,3,46,41$ \\
\hline $\begin{array}{l}\text { F6. } \\
\text { GC18886 }\end{array}$ & $\begin{array}{l}\text { MCOP/ } \\
\text { RP }\end{array}$ & MFRP & c. $1231 \mathrm{~T}>\mathrm{C}$ & $\begin{array}{l}\text { p.(Tyr411 } \\
\text { His) }\end{array}$ & HOM & 10 & $\mathrm{NP}$ & 83 & D & $\begin{array}{l}\text { Prob. } \\
\text { dam. }\end{array}$ & $\mathrm{C} 0$ & $\begin{array}{l}\begin{array}{l}\text { Dis. } \\
\text { caus. }\end{array} \\
\end{array}$ & Absent & Class 3 & This study \\
\hline \multirow[b]{2}{*}{$\begin{array}{l}\text { F7. } \\
\text { GC20271 }\end{array}$} & \multirow[b]{2}{*}{$\begin{array}{l}\text { MCOP/ } \\
\text { RP }\end{array}$} & \multirow[b]{2}{*}{$M F R P$} & c. $955 \mathrm{C}>\mathrm{T}$ & p. $\left(\mathrm{G} \ln 319^{*}\right)$ & HTZ & 8 & I & I & 1 & I & 1 & 1 & Absent & Class 5 & This study \\
\hline & & & $\begin{array}{l}\text { c.6087_54 } \\
\text { +40delins } \\
\text { TAGTGGT }\end{array}$ & none & HTZ & $\begin{array}{l}\text { 5'UTR } \\
\& 1\end{array}$ & I & I & 1 & I & 1 & 1 & Absent & $?$ & This study \\
\hline $\begin{array}{l}\text { F8. } \\
\text { B10315 }\end{array}$ & $\begin{array}{l}\mathrm{NNO/} \\
\mathrm{RP}\end{array}$ & $M F R P$ & c.498del & $\begin{array}{l}\text { p.(Asn167 } \\
\text { Thrfs*25) }\end{array}$ & HOM & 5 & NP & 1 & 1 & 1 & 1 & 1 & $\begin{array}{l}\begin{array}{l}0.0004024 \% \\
(0 \mathrm{HOM})\end{array} \\
\end{array}$ & Class 5 & $36,39,42,48$ \\
\hline $\begin{array}{l}\text { F9. } \\
\text { B16796 }\end{array}$ & MCOP & MFRP & c.1090_1094del & $\begin{array}{l}\text { p.(Thr364 } \\
\text { Glnfs*26) }\end{array}$ & ном & 9 & Yes & I & 1 & I & 1 & 1 & \begin{tabular}{|l|}
$0.0004102 \%$ \\
$(0 \mathrm{HOM})$
\end{tabular} & Class 5 & This study \\
\hline $\begin{array}{l}\text { F10. } \\
\text { B08047 }\end{array}$ & NNO & MFRP & c.498del & $\begin{array}{l}\text { p.(Asn167 } \\
\text { Thrfs*25) }\end{array}$ & ном & 5 & Yes & I & 1 & I & 1 & 1 & $\begin{array}{l}0.0004024 \% \\
(0 \mathrm{HOM})\end{array}$ & Class 5 & $36,39,42,48$ \\
\hline \multirow{2}{*}{$\begin{array}{l}\text { F11. } \\
\text { GC20258 }\end{array}$} & \multirow{2}{*}{ MCOP } & \multirow{2}{*}{ PRSS56 } & c.833dup & $\begin{array}{l}\text { p.(Val279 } \\
\text { Argfs*2) }\end{array}$ & HTZ & 7 & NP & I & 1 & I & 1 & 1 & Absent & Class 5 & 49 \\
\hline & & & c.1571del & $\begin{array}{l}\text { p.(Val525 } \\
\text { Cysfs*55) }\end{array}$ & HTZ & 13 & NP & 1 & 1 & I & 1 & 1 & Absent & Class 5 & This study \\
\hline $\begin{array}{l}\text { F12. } \\
\text { GC18588 }\end{array}$ & MCOP & PRSS56 & c.1066dupC & $\begin{array}{l}\text { p.(Gln356 } \\
\text { Profs*152) }\end{array}$ & HOM & 9 & NP & 1 & 1 & I & 1 & 1 & Absent & Class 5 & $17,51,53$ \\
\hline $\begin{array}{l}\text { F13. } \\
\text { GC16899 }\end{array}$ & MCOP & PRSS56 & c. $320 \mathrm{G}>\mathrm{A}$ & p.(Gly107Glu) & HOM & 4 & NP & 98 & $\mathrm{D}$ & $\begin{array}{l}\text { Poss. } \\
\text { dam. }\end{array}$ & $\mathrm{C} 25$ & $\begin{array}{l}\text { Dis. } \\
\text { caus. }\end{array}$ & \begin{tabular}{|l|}
$0.009375 \%$ \\
$(0 \mathrm{HOM})$
\end{tabular} & Class 3 & This study \\
\hline $\begin{array}{l}\text { F14. } \\
\text { GC19721 }\end{array}$ & MCOP & PRSS56 & \multirow{8}{*}{ c. $1555 \mathrm{G}>\mathrm{C}$} & \multirow{8}{*}{ p.(Gly519Arg) } & HOM & \multirow{8}{*}{-13} & $\mathrm{NP}$ & \multirow{8}{*}{125} & \multirow{8}{*}{ D } & \multirow{8}{*}{$\begin{array}{l}\text { Prob. } \\
\text { dam. }\end{array}$} & \multirow{8}{*}{$\mathrm{C} 0$} & \multirow{8}{*}{$\begin{array}{l}\text { Dis. } \\
\text { caus. }\end{array}$} & \multirow{8}{*}{ Absent } & \multirow{8}{*}{ Class 4} & \multirow{8}{*}{53} \\
\hline $\begin{array}{l}\text { F15. } \\
\text { RXK4624916 }\end{array}$ & \begin{tabular}{|l|}
$\mathrm{MCOP} /$ \\
$\mathrm{RP}$
\end{tabular} & \multirow{5}{*}{ PRSS56 } & & & ном & & \multirow{5}{*}{ Yes } & & & & & & & & \\
\hline $\begin{array}{l}\text { F15. } \\
\text { RXK3297132 }\end{array}$ & $\begin{array}{l}\text { MCOP/ } \\
\text { RP }\end{array}$ & & & & HOM & & & & & & & & & & \\
\hline $\begin{array}{l}\text { F15. } \\
\text { RXK33447157 }\end{array}$ & MCOP & & & & ном & & & & & & & & & & \\
\hline $\begin{array}{l}\text { F15. } \\
\text { RXK3113374 }\end{array}$ & MCOP & & & & ном & & & & & & & & & & \\
\hline $\begin{array}{l}\text { F15. } \\
\text { RXK4633376 }\end{array}$ & MCOP & & & & HOM & & & & & & & & & & \\
\hline $\begin{array}{l}\text { F16. } \\
\text { RXK4941281 }\end{array}$ & MCOP & PRSS56 & & & HOM & & Yes & & & & & & & & \\
\hline $\begin{array}{l}\text { F17. } \\
\text { RXK4985235 }\end{array}$ & MCOP & PRSS56 & & & HOM & & Yes & & & & & & & & \\
\hline $\begin{array}{l}\text { F18. } \\
\text { B05802 }\end{array}$ & MCOP & PRSS56 & c.766T $>C$ & p.(Cys256Arg) & ном & \multirow[t]{2}{*}{7} & Yes & \multirow{2}{*}{180} & \multirow{2}{*}{ D } & \multirow{2}{*}{$\begin{array}{l}\text { Prob. } \\
\text { dam. }\end{array}$} & \multirow{2}{*}{$\mathrm{C} 0$} & \multirow{2}{*}{$\begin{array}{l}\text { Dis. } \\
\text { caus. }\end{array}$} & \multirow{2}{*}{ Absent } & \multirow{2}{*}{ Class 4} & This study \\
\hline $\begin{array}{l}\text { F19. } \\
\text { B01921 }\end{array}$ & NNO & PRSS56 & c.766T $>C$ & p.(Cys256Arg) & HOM & & Yes & & & & & & & & This study \\
\hline $\begin{array}{l}\text { F20. } \\
\text { B03421 }\end{array}$ & NNO & / & 1 & 1 & I & 1 & I & 1 & 1 & 1 & 1 & 1 & 1 & I & 1 \\
\hline $\begin{array}{l}\text { F21. } \\
\text { B07457 }\end{array}$ & NNO & 1 & 1 & 1 & 1 & 1 & I & 1 & 1 & 1 & 1 & 1 & 1 & 1 & 1 \\
\hline
\end{tabular}

Table 2. Variant assessment of the identified MFRP and PRSS56 mutations in 21 unrelated families with NNO or MCOP. Abbreviations used: FAM. PID: Family. Patient ID; HOM: homozygous; HTZ: heterozygous; NNO: isolated nanophthalmos; MCOP: isolated posterior microphthalmia; RP: retinitis pigmentosa; MCOP/ RP: posterior microphthalmia with RP; NNO/RP: nanophthalmos with RP; /: no mutation identified; NP: not performed; D: deleterious; Prob. Dam.: probably damaging; Poss. Dam.: possibly damaging; Dis. Caus.: disease causing; Class 3: uncertain significance; Class 4: likely pathogenic; Class 5: pathogenic. 
A

GC region
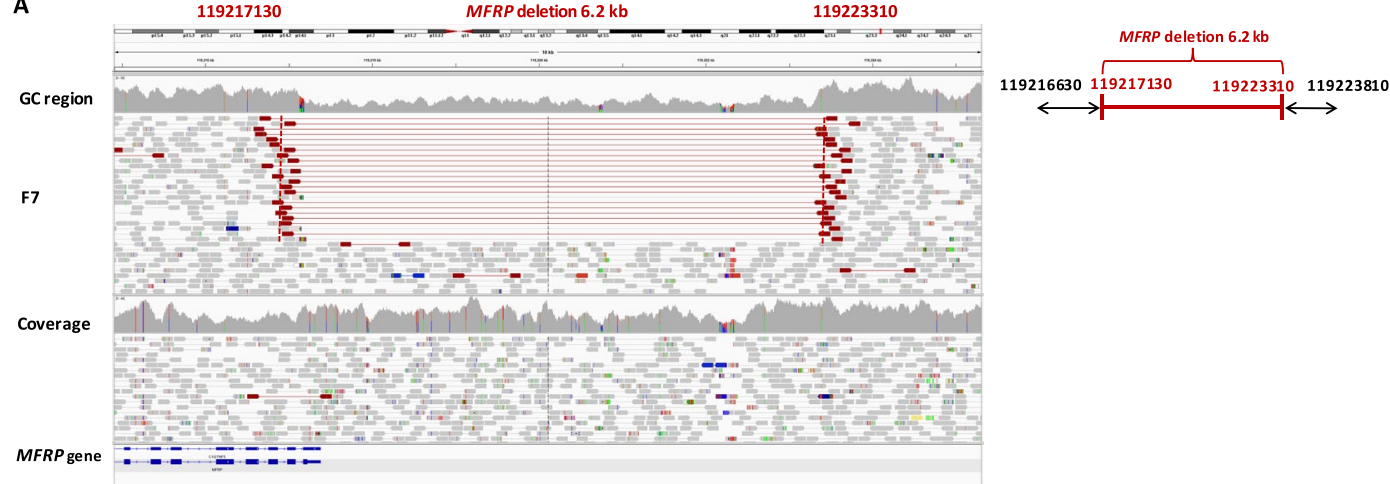

B
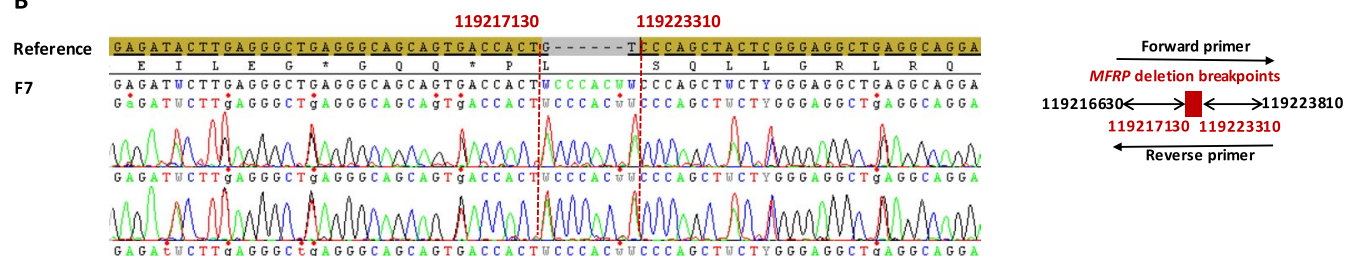

Figure 1. Heterozygous copy number variation implicating $M F R P$ found by whole genome sequencing. The affected patient in family 7 (F7) with MCOP and RP-like changes carries a partial MFRP deletion. (A) Left panel: IGV plot generated by whole genome sequencing, showing a heterozygous deletion of 6.2 kilobases $(\mathrm{kb})$ and an insertion of 7 base pairs (bps) (GRCh37 [hg19] chr11:119, 217, 130_119, 223, 310delinsACCACTA, NM_031433.3 MFRP: c. -6087_54 +40 delinsTAGTGGT; p.?). Highlighted red read pairs have an unusually large insert size suggestive of a large deletion, and the read depth is reduced across the heterozygous deletion. The deletion spans exon 1 of MFRP probably abolishing transcription. Right panel: schematic representation of the deletion. (B) The deletion was confirmed by junction PCR and Sanger sequencing. The arrows in the schematic left panel represent the positions of the primers.

P18), whereas normal responses were found in 4/13 (30.8\%) (P11, P16 and P19 and P22). Representative retinal imaging of these individuals is shown in Fig. 3.

\section{Discussion}

This study characterizes 21 unrelated families with NNO $(\mathrm{n}=6)$ or MCOP $(\mathrm{n}=19)$. Fourteen distinct MFRP and PRSS56 variants were identified in the majority of the studied families (19/21, 90.5\%). Eight different MFRP variants were found in 10/21 (47.6\%) of the families, five of which are novel. Biallelic pathogenic variants were found in 9/10 families, supporting autosomal recessive inheritance. In line with the previously reported 19 distinct $M F R P$ variants, mainly causing the introduction of a premature termination codon ${ }^{20,38-44}$, frameshift and nonsense variants represent the majority of the MFRP mutation spectrum of this study. Some of these variants were found to be recurrent: a novel mutation c.1090_1094del p.(Thr364Glnfs*26) in two unrelated Belgian families (F1 and F9) and a known mutation c.498del p.(Asn167Thrfs*25) in two Moroccan (F2, F10) and one Italian family (F8). The latter variant was previously reported amongst other consanguineous and non-consanguineous families of Spanish, Palestinian, Mexican and Japanese origin ${ }^{9,22,38,42,45,46}$. Interestingly, the previously reported reciprocal duplication c.498dup p.(Asn $167 \mathrm{Glnfs} * 34)^{8,43}$, which might point to a mutational hotspot, was found in a Dagestanian family (F5) in our cohort.

In one individual with MCOP (F4) a monoallelic MFRP variant was found c.491_492insT p.(Asn167Gl$\mathrm{nfs} * 34)^{43}$, with an as yet unidentified second mutation. A structural variant including a copy number variant $(\mathrm{CNV})$, deep intronic mutation of regulatory mutation are possible underlying causes to be explored further. Indeed, this study provided evidence for the occurrence of CNVs affecting MFRP with the identification of the $6.2 \mathrm{~kb}$ deletion c.6087_54 +40delinsTAGTGGT.

To date, nine distinct PRSS56 pathogenic variants have been described, mostly frameshift and nonsense mutations ${ }^{17,24,47,48}$. In our cohort, we detected six different PRSS56 mutations in 9/21 (42.8\%) families, some of which are known as recurrent mutations. Specifically, the previously reported missense variant c.1555G $>$ A p.(Gly519Arg), which was found in a Saudi Arabian family ${ }^{41,48}$, was identified in four unrelated families of Pakistani origin in this study (F14, F15, F16 and F17), suggestive of a founder mutation in this population. Another recurrent mutation is c.1066dup p.(Gln356Profs*152) found in a Pakistani family (F12), which was previously reported in six Tunisian and five Saudi Arabian families ${ }^{17,48,49}$. Furthermore, two novel missense mutations were found in the PRSS56 group in patients with isolated MCOP (F13, F18) and NNO (F19).

Overall, isolated cases with NNO or MCOP were found in $13 / 19(68.4 \%)$ of the families with a molecular diagnosis, mostly in the PRSS56-associated group (11/13, 84.6\%). Complex cases with retinal involvement (RP features or ERG changes) were found in 6/19 (31.6\%) of the families with a molecular diagnosis, mostly due to MFRP mutations $(5 / 6,83.3 \%)$. The retinal involvement in the MFRP-associated group is in line with previous phenotypic studies ${ }^{39}$ and is in agreement with its expression pattern in human, mouse and zebrafish eyes 

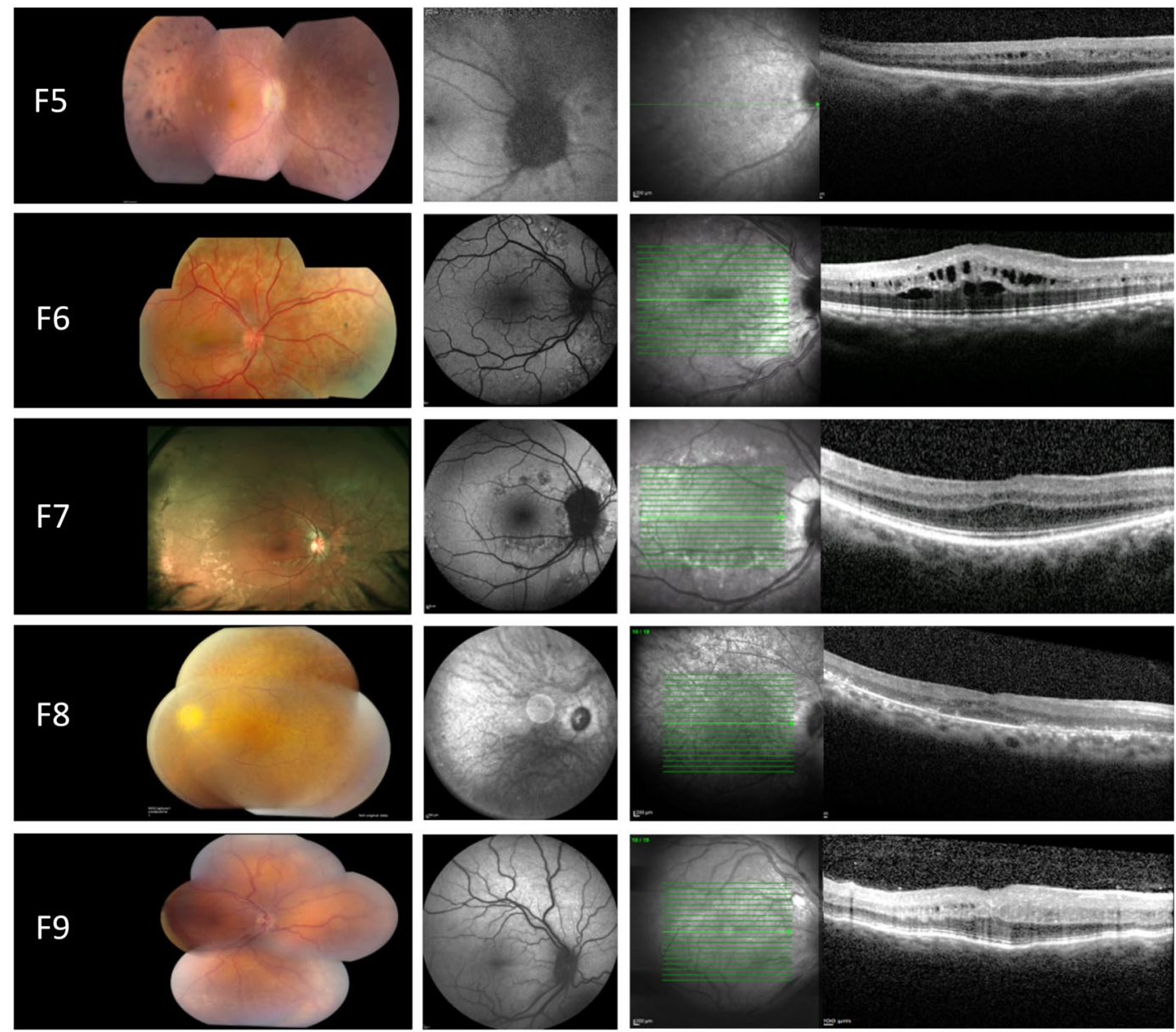

Figure 2. Retinal imaging from the right eye of patients with NNO or MCOP due to mutations in MFRP. Left panel: color fundus. Middle panel: fundus autofluorescence imaging (FAF). Right panel: optical coherence tomography (OCT). F5: color fundoscopy showing crowded optic disc, mid-peripheral intraretinal hyperpigmentation with corresponding hypo-autofluorescence on FAF, foveal hypoplasia and intraretinal cystic cavities on OCT. F6: mid-peripheral hypopigmentary retinal pigment epithelium (RPE) changes with corresponding hyper- and hypo-autofluorescence on fundus autofluorescence imaging (FAF), cystic macular cavities on optical coherence tomography (OCT). F7: posterior pole and mid-peripheral hyper- and hypo-pigmentary RPE change with corresponding hyper- and hypo-autofluorescence on FAF, thickened OCT with foveal hypoplasia. F8: color fundoscopy with crowded optic disc, slight peripheral intraretinal hyperpigmentation and large posterior pole white dots corresponding with hyper- and hypo-autofluorescence on FAF imaging, foveal hypoplasia and cystic macular cavities on OCT. F9: color fundoscopy showing crowded optic disc and normal autofluorescence on FAF imaging, thickened OCT with foveal hypoplasia and occasional intraretinal cyst.

including neural and pigmentary retina ${ }^{3,20,21}$ and its role in photoreceptor outer segment maintenance ${ }^{50}$. An additional explanation could be the fact that MFRP and a gene implicated in late-onset retinal dystrophy, C1QTNF5 (encoding $\mathrm{Clq}$ and tumor necrosis factor related protein 5) are both expressed as a bicistronic transcript and found to co-localize to the same tissues with a clear functional relationship in the retina ${ }^{51,52}$.

The presence of papillomacular folds was found to be more frequent in MCOP $(12 / 19,63.2 \%)$ than in NNO $(3 / 6,50 \%)$. It has been proposed that these papillomacular folds result from the disparity between the retinal and scleral growth which seems to be more prominent in MCOP cases, although no clear genotype-phenotype correlations have been established yet ${ }^{16,21,48}$.

Finally, no clear genotype-phenotype correlations could be established in our studied cohort. For instance in the MFRP group both F2 and F3, having a 'null' allele and a missense variant respectively, displayed posterior microphthalmos without RP-like changes. On the other hand, clinical heterogeneity was observed in the PRSS56 group, illustrated by a missense variant c.766T > C, p.(Cys256Arg) identified in F18 with a clinical diagnosis of MCOP and in F19 with a diagnosis of NNO.

Finally, a definite genetic diagnosis opens up opportunities for gene-based therapies in MFRP-associated retinal disease. Indeed, studies in two mouse models have demonstrated that $M F R P$-retinopathy is a potential target for gene-based therapy: $M f r p^{r d 6} / M f r p^{r d 6}$ described by Dinculescu et al.$^{53}$ and $M f r p K I / K I$ described by Chekuri et al. ${ }^{54}$. 

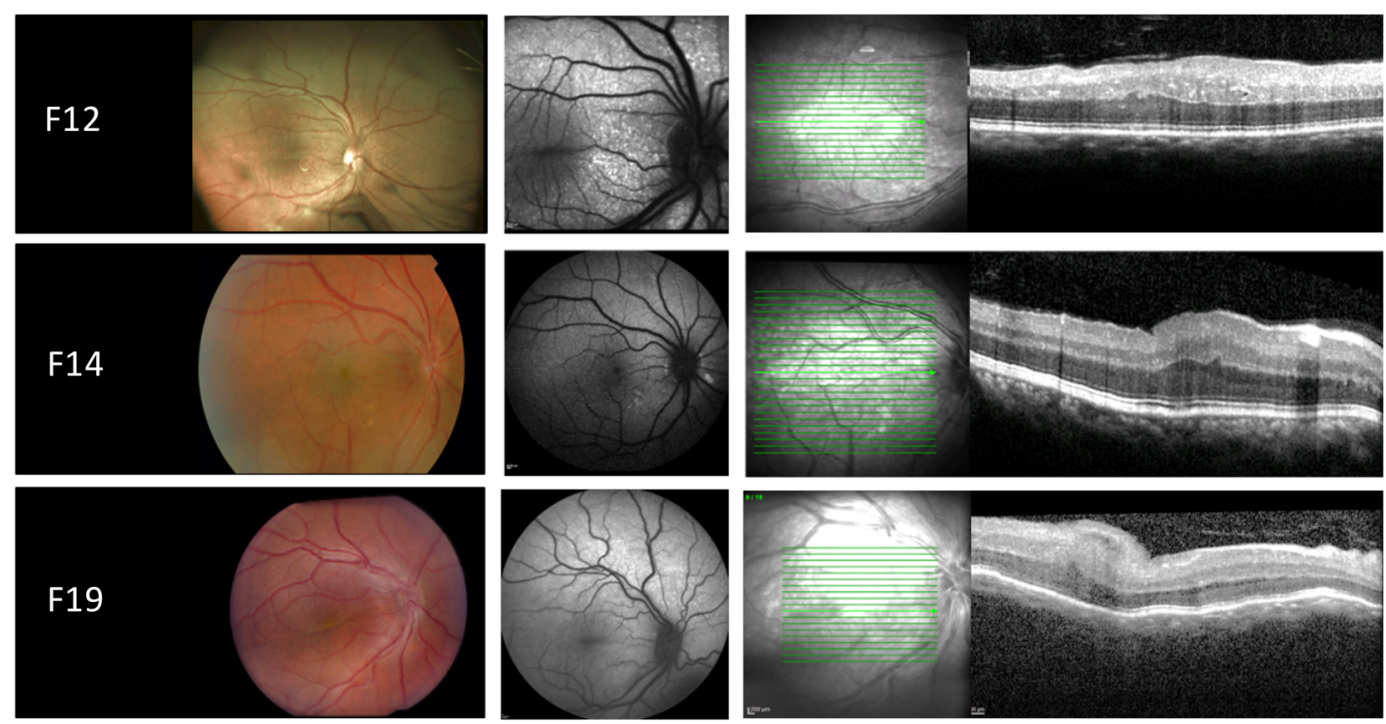

Figure 3. Retinal imaging from the right eye of patients with NNO or MCOP due to mutations in PRSS56. Left panel: color fundus. Middle panel: fundus autofluorescence imaging (FAF). Right panel: optical coherence tomography (OCT). F12: papillomacular fold with diffuse white dots in the posterior pole and to a lesser extent throughout retina, increased autofluorescence at sites of white dots, thickened OCT with occasional intraretinal cyst. F14: papillomacular fold, large posterior pole white dots that have increased autofluorescence, thickened OCT. F19: color fundoscopy showing crowded optic disc and prominent papillomacular fold, normal autofluorescence on FAF imaging, thickened OCT with foveal hypoplasia and papillomacular fold.

In conclusion, MFRP and PRSS56 pathogenic variants, including the first genomic rearrangement of MFRP, were found in the majority $(19 / 21,90.5 \%)$ of the studied families, displaying a large phenotypic variability. No mutations were found in two Belgian families with NNO, leaving the possibility to identify underlying mutations in other NNO genes, or to uncover (a) novel NNO gene(s). Overall, this study expands the phenotypic and molecular spectrum of MFRP- and PRSS56-associated autosomal recessive NNO and MCOP in the largest cohort reported to date.

Received: 1 March 2019; Accepted: 9 December 2019;

Published online: 28 January 2020

\section{References}

1. Warburg, M. Classification of microphthalmos and coloboma. Journal of medical genetics 30, 664-669, https://doi.org/10.1136/ jmg.30.8.664 (1993).

2. Elder, M. J. Aetiology of severe visual impairment and blindness in microphthalmos. The British journal of ophthalmology 78, 332-334, https://doi.org/10.1136/bjo.78.5.332 (1994).

3. Sundin, O. H. et al. Developmental basis of nanophthalmos: MFRP Is required for both prenatal ocular growth and postnatal emmetropization. Ophthalmic Genet 29, 1-9, https://doi.org/10.1080/13816810701651241 (2008).

4. Kallen, B. \& Tornqvist, K. The epidemiology of anophthalmia and microphthalmia in Sweden. European journal of epidemiology 20, 345-350, https://doi.org/10.1007/s10654-004-6880-1 (2005).

5. Verma, A. S. \& Fitzpatrick, D. R. Anophthalmia and microphthalmia. Orphanet journal of rare diseases 2, 47, https://doi. org/10.1186/1750-1172-2-47 (2007).

6. Auffarth, G. U., Blum, M., Faller, U., Tetz, M. R. \& Volcker, H. E. Relative anterior microphthalmos: morphometric analysis and its implications for cataract surgery. Ophthalmology 107, 1555-1560, https://doi.org/10.1016/s0161-6420(00)00240-2 (2000).

7. Wu, W. et al. Cataract surgery in patients with nanophthalmos: results and complications. Journal of cataract and refractive surgery 30, 584-590, https://doi.org/10.1016/j.jcrs.2003.07.009 (2004).

8. Ayala-Ramirez, R. et al. A new autosomal recessive syndrome consisting of posterior microphthalmos, retinitis pigmentosa, foveoschisis, and optic disc drusen is caused by a MFRP gene mutation. Molecular vision 12, 1483-1489 (2006).

9. Zenteno, J. C., Buentello-Volante, B., Quiroz-Gonzalez, M. A. \& Quiroz-Reyes, M. A. Compound heterozygosity for a novel and a recurrent MFRP gene mutation in a family with the nanophthalmos-retinitis pigmentosa complex. Molecular vision 15, 1794-1798 (2009).

10. Sonmez, K. \& Ozcan, P. Y. Angle-closure glaucoma in a patient with the nanophthalmos-ocular cystinosis-foveoschisis-pigmentary retinal dystrophy complex. BMC ophthalmology 12, 23, https://doi.org/10.1186/1471-2415-12-23 (2012).

11. Fuchs, J. et al. Hereditary high hypermetropia in the Faroe Islands. Ophthalmic genetics 26, 9-15, https://doi. org/10.1080/13816810590918406 (2005).

12. Cross, H. E. \& Yoder, F. Familial nanophthalmos. American journal of ophthalmology 81, 300-306, https://doi.org/10.1016/00029394(76)90244-0 (1976).

13. Walsh, M. K. \& Goldberg, M. F. Abnormal foveal avascular zone in nanophthalmos. American journal of ophthalmology 143, 1067-1068, https://doi.org/10.1016/j.ajo.2007.01.051 (2007).

14. Sarvananthan, N. et al. The prevalence of nystagmus: the Leicestershire nystagmus survey. Investigative ophthalmology \& visual science 50, 5201-5206, https://doi.org/10.1167/iovs.09-3486 (2009).

15. Zacharias, L. C. et al. Efficacy of topical dorzolamide therapy for cystoid macular edema in a patient with MFRP-related nanophthalmos-retinitis pigmentosa-foveoschisis-optic disk drusen syndrome. Retin Cases Brief Rep 9, 61-63, https://doi. org/10.1097/ICB.0000000000000088 (2015). 
16. Serrano, J. C., Hodgkins, P. R., Taylor, D. S., Gole, G. A. \& Kriss, A. The nanophthalmic macula. The British journal of ophthalmology 82, 276-279, https://doi.org/10.1136/bjo.82.3.276 (1998).

17. Gal, A. et al. Autosomal-recessive posterior microphthalmos is caused by mutations in PRSS56, a gene encoding a trypsin-like serine protease. American journal of human genetics 88, 382-390, https://doi.org/10.1016/j.ajhg.2011.02.006 (2011).

18. Albar, A. A., Nowilaty, S. R. \& Ghazi, N. G. Posterior microphthalmos and papillomacular fold-associated cystic changes misdiagnosed as cystoid macular edema following cataract extraction. Clinical ophthalmology (Auckland, N.Z.) 9, 73-76, https://doi. org/10.2147/OPTH.S75771 (2015).

19. Katoh, M. Molecular cloning and characterization of MFRP, a novel gene encoding a membrane-type Frizzled-related protein. Biochemical and biophysical research communications 282, 116-123, https://doi.org/10.1006/bbrc.2001.4551 (2001).

20. Sundin, O. H. et al. Extreme hyperopia is the result of null mutations in MFRP, which encodes a Frizzled-related protein. Proc Natl Acad Sci USA 102, 9553-9558, https://doi.org/10.1073/pnas.0501451102 (2005).

21. Collery, R. F., Volberding, P. J., Bostrom, J. R., Link, B. A. \& Besharse, J. C. Loss of Zebrafish Mfrp Causes Nanophthalmia, Hyperopia, and Accumulation of Subretinal Macrophages. Investigative ophthalmology \& visual science 57, 6805-6814, https://doi. org/10.1167/iovs.16-19593 (2016)

22. Neri, A. et al. Membrane frizzled-related protein gene-related ophthalmological syndrome: 30 -month follow-up of a sporadic case and review of genotype-phenotype correlation in the literature. Molecular vision 18, 2623-2632 (2012).

23. Soundararajan, R. et al. Gene profiling of postnatal Mfrprd6 mutant eyes reveals differential accumulation of Prss56, visual cycle and phototransduction mRNAs. PloS one 9, e110299, https://doi.org/10.1371/journal.pone.0110299 (2014).

24. Nair, K. S. et al. Alteration of the serine protease PRSS56 causes angle-closure glaucoma in mice and posterior microphthalmia in humans and mice. Nature genetics 43, 579-584, https://doi.org/10.1038/ng.813 (2011).

25. Kiefer, A. K. et al. Genome-wide analysis points to roles for extracellular matrix remodeling, the visual cycle, and neuronal development in myopia. PLoS genetics 9, e1003299, https://doi.org/10.1371/journal.pgen.1003299 (2013).

26. Verhoeven, V. J. et al. Genome-wide meta-analyses of multiancestry cohorts identify multiple new susceptibility loci for refractive error and myopia. Nature genetics 45, 314-318, https://doi.org/10.1038/ng.2554 (2013).

27. McCulloch, D. L. et al. ISCEV Standard for full-field clinical electroretinography (2015 update). Documenta ophthalmologica. Advances in ophthalmology 130, 1-12, https://doi.org/10.1007/s10633-014-9473-7 (2015).

28. Purcell, S. et al. PLINK: a tool set for whole-genome association and population-based linkage analyses. American journal of human genetics 81, 559-575, https://doi.org/10.1086/519795 (2007).

29. Sante, T. et al. ViVar: a comprehensive platform for the analysis and visualization of structural genomic variation. PloS one $\mathbf{9}$, e113800, https://doi.org/10.1371/journal.pone.0113800 (2014).

30. Coppieters, F. et al. Massively parallel sequencing for early molecular diagnosis in Leber congenital amaurosis. Genetics in medicine: official journal of the American College of Medical Genetics 14, 576-585, https://doi.org/10.1038/gim.2011.51 (2012).

31. Raczy, C. et al. Isaac: ultra-fast whole-genome secondary analysis on Illumina sequencing platforms. Bioinformatics (Oxford, England) 29, 2041-2043, https://doi.org/10.1093/bioinformatics/btt314 (2013).

32. Carss, K. J. et al. Comprehensive Rare Variant Analysis via Whole-Genome Sequencing to Determine the Molecular Pathology of Inherited Retinal Disease. Am J Hum Genet 100, 75-90, https://doi.org/10.1016/j.ajhg.2016.12.003 (2017).

33. Hull, S. et al. Nonsyndromic Retinal Dystrophy due to Bi-Allelic Mutations in the Ciliary Transport Gene IFT140. Invest Ophthalmol Vis Sci 57, 1053-1062, https://doi.org/10.1167/iovs.15-17976 (2016).

34. Richards, S. et al. Standards and guidelines for the interpretation of sequence variants: a joint consensus recommendation of the American College of Medical Genetics and Genomics and the Association for Molecular Pathology. Genet Med 17, 405-424, https:// doi.org/10.1038/gim.2015.30 (2015).

35. Vissers, L. E. et al. Rare pathogenic microdeletions and tandem duplications are microhomology-mediated and stimulated by local genomic architecture. Hum Mol Genet 18, 3579-3593, https://doi.org/10.1093/hmg/ddp306 (2009).

36. Verdin, H. et al. Microhomology-mediated mechanisms underlie non-recurrent disease-causing microdeletions of the FOXL2 gene or its regulatory domain. PLoS Genet 9, e1003358, https://doi.org/10.1371/journal.pgen.1003358 (2013).

37. Zhang, F., Gu, W., Hurles, M. E. \& Lupski, J. R. Copy number variation in human health, disease, and evolution. Annu Rev Genomics Hum Genet 10, 451-481, https://doi.org/10.1146/annurev.genom.9.081307.164217 (2009).

38. Crespi, J. et al. A novel mutation confirms MFRP as the gene causing the syndrome of nanophthalmos-renititis pigmentosafoveoschisis-optic disk drusen. Am J Ophthalmol 146, 323-328, https://doi.org/10.1016/j.ajo.2008.04.029 (2008).

39. Mukhopadhyay, R. et al. A detailed phenotypic assessment of individuals affected by MFRP-related oculopathy. Mol Vis 16, 540-548 (2010).

40. Pehere, N., Jalali, S., Deshmukh, H. \& Kannabiran, C. Posterior microphthalmos pigmentary retinopathy syndrome. Doc Ophthalmol 122, 127-132, https://doi.org/10.1007/s10633-011-9266-1 (2011).

41. Aldahmesh, M. A. et al. Posterior microphthalmos as a genetically heterogeneous condition that can be allelic to nanophthalmos. Arch Ophthalmol 129, 805-807, https://doi.org/10.1001/archophthalmol.2011.129 (2011).

42. Matsushita, I., Kondo, H. \& Tawara, A. Novel compound heterozygous mutations in the MFRP gene in a Japanese patient with posterior microphthalmos. Jpn J Ophthalmol 56, 396-400, https://doi.org/10.1007/s10384-012-0145-4 (2012).

43. Wasmann, R. A. et al. Novel membrane frizzled-related protein gene mutation as cause of posterior microphthalmia resulting in high hyperopia with macular folds. Acta Ophthalmol 92, 276-281, https://doi.org/10.1111/aos.12105 (2014).

44. Xu, Y. et al. Identification of MFRP Mutations in Chinese Families with High Hyperopia. Optom Vis Sci 93, 19-26, https://doi. org/10.1097/OPX.0000000000000751 (2016).

45. Beryozkin, A. et al. Identification of mutations causing inherited retinal degenerations in the israeli and palestinian populations using homozygosity mapping. Invest Ophthalmol Vis Sci 55, 1149-1160, https://doi.org/10.1167/iovs.13-13625 (2014).

46. Richardson, R., Tracey-White, D., Webster, A. \& Moosajee, M. The zebrafish eye-a paradigm for investigating human ocular genetics. Eye (Lond) 31, 68-86, https://doi.org/10.1038/eye.2016.198 (2017).

47. Orr, A. et al. Mutations in a novel serine protease PRSS56 in families with nanophthalmos. Mol Vis 17, 1850-1861 (2011).

48. Nowilaty, S. R. et al. Biometric and molecular characterization of clinically diagnosed posterior microphthalmos. Am J Ophthalmol 155, 361-372 e367, https://doi.org/10.1016/j.ajo.2012.08.016 (2013).

49. Jiang, D. et al. Evaluation of PRSS56 in Chinese subjects with high hyperopia or primary angle-closure glaucoma. Mol Vis 19, 2217-2226 (2013).

50. Won, J. et al. Membrane frizzled-related protein is necessary for the normal development and maintenance of photoreceptor outer segments. Vis Neurosci 25, 563-574, https://doi.org/10.1017/S0952523808080723 (2008).

51. Mandal, M. N. et al. Spatial and temporal expression of MFRP and its interaction with CTRP5. Invest Ophthalmol Vis Sci 47, 5514-5521, https://doi.org/10.1167/iovs.06-0449 (2006).

52. Fogerty, J. \& Besharse, J. C. 174delG mutation in mouse MFRP causes photoreceptor degeneration and RPE atrophy. Invest Ophthalmol Vis Sci 52, 7256-7266, https://doi.org/10.1167/iovs.11-8112 (2011).

53. Dinculescu, A. et al. Gene therapy for retinitis pigmentosa caused by MFRP mutations: human phenotype and preliminary proof of concept. Hum Gene Ther 23, 367-376, https://doi.org/10.1089/hum.2011.169 (2012).

54. Chekuri, A. et al. Long-Term Effects of Gene Therapy in a Novel Mouse Model of Human MFRP-Associated Retinopathy. Hum Gene Ther 30, 632-650, https://doi.org/10.1089/hum.2018.192 (2019). 


\section{Acknowledgements}

This study was approved and funded by grants from the Research Foundation Flanders (FWO, Brussels, Belgium) to E.D.B. and B.P.L. who are FWO Senior Clinical Investigators (1802220N and 1803816N), and King Saud University (Riyadh, Saudi Arabia) to B.A. E.D.B. and B.P.L. are members of ERN-EYE. This study was supported by the Ghent University Special Research Fund (BOF15/GOA/011) to E.D.B., Hercules Foundation AUGE/13/023 to E.D.B., by Funds for Research in Ophthalmology (FRO) to B.A. NIHR-Biomedical Research Centre at Moorfields Eye Hospital and UCL Institute of Ophthalmology, Fight for Sight, RP Fighting Blindness, The Rosetrees Trust, Moorfields Special Trustees. G.A. is supported by a Fight for Sight Early Career Investigator award. The authors are very grateful to the families who participated in this study.

\section{Author contributions}

B.A. performed the genetic analyses, the experiments and data analysis, and drafted the initial manuscript. G.A. performed the genetic analyses, the experiments and data analysis, and critically reviewed and revised the manuscript. H.V. assisted with the data analysis, and critically reviewed and revised the manuscript. J.D.Z., I.B., I.C., T.D.R., S.H., M.S., A.D., M.P., D.W., J.R.A., A.R.W., B.P.L. and A.T.M. managed patients, provided and interpreted clinical data and critically reviewed the manuscript. E.D.B. conceptualized the study, supervised genetic analyses and critically reviewed and revised the manuscript. All authors provided critical input and approved the final manuscript as submitted.

\section{Competing interests}

The authors declare no competing interests.

\section{Additional information}

Supplementary information is available for this paper at https://doi.org/10.1038/s41598-019-57338-2.

Correspondence and requests for materials should be addressed to E.D.B.

Reprints and permissions information is available at www.nature.com/reprints.

Publisher's note Springer Nature remains neutral with regard to jurisdictional claims in published maps and institutional affiliations.

(c) (1) Open Access This article is licensed under a Creative Commons Attribution 4.0 International License, which permits use, sharing, adaptation, distribution and reproduction in any medium or format, as long as you give appropriate credit to the original author(s) and the source, provide a link to the Creative Commons license, and indicate if changes were made. The images or other third party material in this article are included in the article's Creative Commons license, unless indicated otherwise in a credit line to the material. If material is not included in the article's Creative Commons license and your intended use is not permitted by statutory regulation or exceeds the permitted use, you will need to obtain permission directly from the copyright holder. To view a copy of this license, visit http://creativecommons.org/licenses/by/4.0/.

(C) The Author(s) 2020 